\title{
LINEARLY ORDERED TOPOLOGICAL SPACES
}

\section{S. L. GULdEN, W. M. FLEISCHMAN AND J. H. WESTON}

This work is devoted to the study of certain cardinality modifications of paracompactness and compactness in the setting of linearly ordered spaces. Some of the concepts treated here have previously been studied by Aquaro [1] ${ }^{1}$, Gulden [4], Kennison [5], Mansfield [6], Morita [7], and Poppe [9]. On the other hand, the concept of $\mathrm{m}$-boundedness, introduced in $\$ 2$, is new.

Our main results (Theorems 1 and 3 ) establish the equivalence for linearly ordered spaces of a number of cardinality modifications of, in the first case, paracompactness, and, in the second, compactness. In each instance, this is accomplished by means of a characterization in terms of conditions imposed on the gaps of the space. In regard to Theorem 1, in which the concept of $Q$-gap introduced by Gillman and Henriksen [3] plays a crucial role, we call attention to the equivalence of m-paracompactness and the apparently much stronger condition $m$-full normality in the setting of linearly ordered spaces. It is also of interest to note that Theorem 3 shows the equivalence of $\mathfrak{m}$-compactness and $\mathfrak{m}$-boundedness, again in the setting of linearly ordered spaces. Novak [8] has shown this latter equivalence is not in general true for $m$ countable, but the authors are not aware of an $\mathfrak{m}$-compact space which is not $\mathfrak{m}$-bounded for $\mathfrak{m}$ larger than countable.

1. In this section, we note the equivalence, in the setting of linearly ordered spaces, of a varied collection of cardinality modifications of paracompactness. Unless otherwise indicated, $m$ will denote an infinite cardinal.

Definition 1. The space $X$ is said to be $\mathfrak{m}$-paracompact ( $\mathfrak{m}$-metacompact, strongly m-paracompact) if and only if each open covering of $X$ by no more than $m$ sets admits as a refinement a locally finite (point finite, star finite) open covering.

Definition 2 (Mansfield [6]). (Here, let $\mathfrak{m}$ be any cardinal $\geqq 2$.) Let $Q$ and $B$ be collections of subsets of a set $X$. $B$ is called an $m$-star (almost $\mathfrak{m}$-star) refinement of $Q$ if and only if $B$ refines $a$ and whenever $\mathfrak{N} \subseteq \emptyset$ with $|\mathfrak{T}| \leqq \mathfrak{m}$ and $\cap \mathscr{T} \neq \varnothing(M \subseteq X$ with $|M| \leqq \mathfrak{m}$ and $M \subseteq \operatorname{St}(x, \beta)$ for some $x \in X)$ there is an $A \in Q$ with $U \mathscr{M} \subseteq A(M \subseteq A)$. The space $X$ is $\mathfrak{m}$-fully normal (almost $\mathrm{m}$-fully normal) if and only if to each open covering of $X$ there corresponds an open covering which $\mathfrak{m}$-star (almost $\mathrm{m}$-star) refines it.

Received by the editors January 8, 1969.

${ }^{1}$ Numbers in brackets refer to the list of references at the end of this paper. 
It is clear that for any $m \geqq 2$, almost $m$-fully normality is implied by $\mathfrak{m}$-full normality; also, if $\mathfrak{n} \geqq \mathfrak{m}$ then (almost) $\mathfrak{n}$-full normality implies (almost) m-full normality. Mansfield's result that every linearly ordered space is $\boldsymbol{\aleph}_{0}$-fully normal will be seen to be a corollary of our Theorem 1. On the other hand, the proofs of the implications (ix) $\Rightarrow$ (i), (ix) $\Rightarrow$ (ii) of Theorem 1 are based upon Mansfield's proof of the result cited. In particular, we make use of the following lemma [6, Lemma 3.1].

Lemma 1. Let $X$ be a linearly ordered space (that is, a linearly ordered set provided with its interval topology) with the property that every strictly increasing (decreasing) sequence in $X$ indexed by the positive integers, with their usual order, converges to a point of $X$. Then for each open covering $u$ of $X$ there is a point $u^{*} \in X$ such that $\left\{x \in X \mid x>u^{*}\right\}$ $\subseteq \operatorname{St}\left(u^{*}, \mathfrak{u}\right)\left(\left\{x \in X \mid x<u^{*}\right\} \subseteq \operatorname{St}\left(u^{*}, \mathfrak{u}\right)\right)$.

Morita [7, Theorem 4.2] has shown that for any infinite cardinal $\mathfrak{m}$, almost $\mathfrak{m}$-full normality implies $\mathfrak{m}$-paracompactness. The same argument, suitably modified, may be used to demonstrate that strong $\mathrm{m}$-paracompactness is implied by the property given as (ii) in Theorem 1 below. This property itself came to light as a result of an observation of Aquaro.

Definition 3 (Aquaro [1]). Let $\propto$ and $\beta$ be collections of subsets of a set $X$. $B$ is said to be an $m$-quasi-refinement of $Q$ if and only if for any $M \subseteq X$ with $|M| \leqq \mathfrak{m}$, if $M \subseteq B$ for some $B \in B$ then there is an $A \in Q$ such that $M \subseteq A$.

Aquaro observed that a normal space is almost $m$-fully normal if and only if to each open covering of the space there corresponds a locally finite open covering which m-quasi-refines it.

The concept of $Q$-gap, introduced by Gillman and Henriksen, is that by which the cardinality modifications of paracompactness under discussion are ultimately related.

Definition 4 (Gillman and Henriksen [3]). Let $X$ be a linearly ordered set and let $X^{+}$denote the order completion of $X$. Let $\omega_{\alpha}$ and $\omega_{\beta}$ be regular initial ordinals and let $\omega_{\beta}^{*}$ denote $\omega_{\beta}$ with the reverse order. A gap $u$ of $X$ (i.e., an element of $X^{+} \backslash X$ ) is an $\omega_{\alpha}$-limit of $X$ (an $\omega_{\beta}{ }^{*}$-limit of $X$ ) if the set of all elements of $X$ which precede $u$ (which follow $u$ ) is cofinal (coinitial) with $\omega_{\alpha}$ (with $\omega_{\beta}^{*}$ ). The unique ordinal for which $u$ is an $\omega_{\alpha}$-limit of $X$ (an $\omega_{\beta}^{*}$-limit of $X$ ) will be denoted by $\omega_{\alpha(u)}$ (by $\omega_{\beta(u)}$ ). (By $S$ is cofinal with $\omega_{\alpha}$ we mean $S$ has an unbounded subset which is well ordered of order type $\omega_{\alpha}$.)

A gap $u$ of $X$ is called a $Q$-gap from the left (right) if and only if there is a regular initial ordinal $\omega_{\alpha}$ and an increasing (decreasing) sequence 
$\left\{x_{\beta} \mid \beta<\omega_{\alpha}\right\}$ of points of $X^{+}$such that $u=\lim _{\beta<\omega_{a}} x_{\beta}$ and if $\lambda<\omega_{\alpha}$ is any nonzero limit ordinal then $\lim _{\beta<\lambda} x_{\beta}$ is a gap of $X$. (Thus any gap which is the limit of an increasing (decreasing) sequence of points of $X^{+}$indexed by the positive integers is a $Q$-gap from the left (right).)

It was proved in [3] that a linearly ordered space is paracompact if and only if each of its gaps is a $Q$-gap from both right and left (with the obvious modification for the possible endgaps of the space). In a recent work [2], Fedorchuk has shown that in linearly ordered spaces, strong paracompactness, paracompactness, and metacompactness are mutually equivalent.

The following lemma (9.4 of [3]) is of use in the proof of Theorem 1.

Lemma 2. Let $J=(p, v)$ be an interval of a linearly ordered space $X$, where $v$ is a gap that is not a $Q$-gap from the left. Let $\alpha$ be the ordinal for which $v$ is an $\omega_{\alpha}$-limit. Let $\mathcal{u}$ be an open covering of $J$ that does not cover the gap v. Then $u$ has a subfamily of power $\left|\omega_{\alpha}\right|$ with nonvoid intersection.

TheOREM 1. Let $X$ be a linearly ordered space, and let $m$ be an infinite cardinal. The following are then equivalent:

(i) $X$ is m-fully normal.

(ii) To each open covering $u$ of $X$ there corresponds a star-finite open covering $\mathcal{V}$ which is an $\mathrm{m}$-quasi-refinement of $\mathcal{u}$.

(iii) $X$ is almost $\mathfrak{m}$-fully normal.

(iv) $X$ is strongly-m-paracompact.

(v) $X$ is $\mathfrak{m}$-paracompact.

(vi) $X$ is $\mathrm{m}$-metacompact.

(vii) Each open covering $u$ of $X$ with $|\mathfrak{u}| \leqq \mathfrak{m}$ admits as a refinement an open covering $V$ which is point countable (that is, no point of $X$ belongs to more than countably many members of $V$ ).

(viii) For each open covering $\mathcal{U}$ of $X$ with $\boldsymbol{N}_{0} \leqq|\mathcal{u}| \leqq m$ there is an open covering $v$ which refines $U$ such that each point of $X$ belongs to less than $|\mathcal{u}|$ members of $v$.

(ix) Each gap u of $X$ satisfying $\left|\omega_{\alpha(u)}\right| \leqq \mathfrak{m}$ (respectively, $\left.\left|\omega_{\beta(u)}\right| \leqq \mathfrak{m}\right)$ is a Q-gap from the left (respectively, right).

Proof. The scheme is (i) $\Rightarrow$ (iii) $\Rightarrow(v)$, (ii) $\Rightarrow$ (iv) $\Rightarrow(v) \Rightarrow(v i) \Rightarrow(v i i)$ $\Rightarrow$ (ix), (vi) $\Rightarrow$ (viii) $\Rightarrow$ (ix), (ix) $\Rightarrow$ (i), (ix) $\Rightarrow$ (ii). Of these implications all save (vii) $\Rightarrow$ (ix), (viii) $\Rightarrow$ (ix), (ix) $\Rightarrow$ (i), and (ix) $\Rightarrow$ (ii) hold in arbitrary spaces.

The implications (vii) $\Rightarrow$ (ix) and (viii) $\Rightarrow$ (ix) follow immediately from Lemma 2.

The proofs of (ix) $\Rightarrow$ (i) and (ix) $\Rightarrow$ (ii) are quite similar. We sketch the latter. We shall say that a gap $u$ of $X$ is covered by the interval 
$(a, b)=\{x \in X \mid a<x<b\} \quad$ (where $\left.a, b \in X^{+}\right)$if and only if, in $X^{+}$, $a<u<b$ or $u$ is an endgap of $X$ and coincides with either $a$ or $b$.

Assume (ix) and let $\mathcal{u}$ be an open covering of $X$. We may suppose that $\mathcal{U}$ is a covering of $X$ by intervals. The set $F^{+}$of gaps of $X$ not covered by any member of $\mathcal{U}$ is a closed subset of $X^{+}$, so that $X^{+} \backslash F^{+}$ can be expressed as the union, $\bigcup_{\alpha \in \Gamma} K_{\alpha}^{+}$, of pairwise disjoint intervals, open in $X^{+}$, whose endpoints are either endpoints of $X^{+}$or gaps of $X$ not covered by any member of $\mathcal{u}$. For each $U=(a, b) \in \mathcal{U}$, let $U^{+}$ $=\left\{z \in X^{+} \mid a<z<b\right\}$. It will suffice to show that for each $\alpha \in \Gamma$, there is a star finite open covering ${\vartheta_{\alpha}^{+}}^{+}$of $K_{\alpha}^{+}$which is an m-quasi-refinement of $u_{\alpha}^{+}=\left\{U^{+} \cap K_{\alpha}^{+} \mid U \in \mathcal{u}\right\}$, for then, if $v_{\alpha}=\left\{V^{+} \cap X \mid V_{\alpha}{ }^{+} \in V^{+}\right\}$and $\mathcal{V}=U\left\{\mathcal{V}_{\alpha} \mid \alpha \in \Gamma\right\}, \vartheta$ will be a star finite open covering of $X$ which is an $\mathfrak{m}$-quasi-refinement of $\mathcal{u}$.

$K_{\alpha}^{+}$is a linearly ordered space with no interior gaps. Thus we distinguish the following cases:

I. $K_{\alpha}^{+}=(u, v)$ where $u$ and $v$ are gaps of $X$ which are $Q$-gaps from the right and left, respectively, or $K_{\alpha}^{+}=[u, v)$ where $u$ is an endpoint of $X^{+}$which is covered by a member of $u$ and $v$ is a $Q$-gap from the left, or $K_{\alpha}^{+}=(u, v]$ where $u$ is a $Q$-gap from the right and $v$ is an endpoint of $X^{+}$covered by some member of $\mathcal{u}$. In each of these instances, application of the results of Gillman and Henriksen, and Fedorchuk cited above shows that $K_{\alpha}^{+}$is strongly paracompact. Thus, the open covering $\mathcal{u}_{\alpha}^{+}$of $K_{\alpha}^{+}$admits as a refinement a star finite open covering $v_{\alpha}^{+}$. Certainly $v_{\alpha}^{+}$is an m-quasi-refinement of $u_{\alpha}^{+}$.

II. $K_{\alpha}^{+}=(u, v)$ where $u$ and $v$ are gaps of $X$, neither of which is covered by any member of $u$, such that $u$ is coinitial with no subset of $X$ of cardinality $\leqq \mathfrak{m}$, and $v$ is cofinal with no subset of $X$ of cardinality $\leqq \mathfrak{m}$. Since $K_{\alpha}^{+}$has no interior gaps, and since $\mathfrak{m}$ is infinite, Lemma 1 guarantees the existence of points $u^{*}, v^{*} \in K_{\alpha}^{+}$such that $U_{L}=\left\{y \in K_{\alpha}^{+} \mid y<u^{*}\right\} \subseteq \operatorname{St}\left(u^{*}, u_{\alpha}^{+}\right)$and $U_{R}=\left\{z \in K_{\alpha}^{+} \mid v^{*}<z\right\}$ $\subseteq \operatorname{St}\left(v^{*}, u_{\alpha}^{+}\right)$. We may assume that $u^{*}<v^{*}$. The subspace $\left[u^{*}, v^{*}\right]$ of $K_{\alpha}^{+}$, having no gaps, is compact. Thus there is a finite collection $\mathcal{u}_{0 \alpha}^{+} \subseteq \mathcal{U}_{\alpha}^{+}$which covers $\left[u^{*}, v^{*}\right] .^{y}$ Let $v_{\alpha}^{+}=\mathcal{u}_{\alpha 0}^{+} \cup\left\{U_{L}, U_{R}\right\}$. Then $v_{\alpha}^{+}$ is a finite (hence star finite) open covering of $K_{\alpha}^{+}$. To establish that $v_{\alpha}^{+}$is an $m$-quasi-refinement of $u_{\alpha}^{+}$it is clearly sufficient to show that if $M \subseteq K_{\alpha}^{+},|M| \leqq \mathfrak{m}$, and $M$ is contained in either of $U_{L}$ or $U_{R}$ then there is a $U \in \mathcal{U}$ such that $M \subseteq U^{+} \cap K_{\alpha}^{+}$. Suppose $M \subseteq U_{R}$. The element $z=\sup M$ of $X^{+}$is cofinal with a subset of $X$ of cardinality $\leqq m$. Thus $v^{*}<z<v$ so that $z \in \operatorname{St}\left(v^{*}, \mathcal{u}_{\alpha}^{+}\right)$. There is a $U \in \mathcal{u}$ such that $v^{*}$, $Z \in U^{+} \cap K_{\alpha}^{+}$. But the members of $\mathcal{U}$ are intervals and for each $y \in M$, $v^{*}<y \leqq z$, so that $M \subseteq U^{+} \cap K_{\alpha}^{+}$. (We remark that the analogous argument for the implication (ix) $\Rightarrow(\mathrm{i})$ follows that of Mansfield [6] in the proof of case I of Lemma 3.5.) 
III. $K_{\alpha}^{+}=(u, v)$ where $u$ is as in II and $v$ is a $Q$-gap from the left (or with the roles of $u$ and $v$ reversed). Choose $u^{*}$ as above. The subspace $\left[u^{*}, v\right)$ is paracompact, hence strongly paracompact.

IV. $K_{\alpha}^{+}=(u, v]$ where $u$ is as in II and $v$ is a covered endpoint of $X^{+}$(or with the roles of $u$ and $v$ reversed). Choose $u^{*}$ as above. The subspace $\left[u^{*}, v\right]$ is compact.

2. In this section we turn our attention from cardinality modifications of paracompactness to similar modifications of compactness. The main result (Theorem 3) shows the equivalence of these compactness modifications in linearly ordered spaces.

Definition 5. Let $X$ be a topological space, and let $m$ be an infinite cardinal. $X$ is called m-bounded if and only if for each $A \subseteq X$ with $|A| \leqq \mathfrak{m}$ there is a compact set $C \subseteq X$ such that $A \subseteq C . X$ is called $\mathfrak{m}$-compact if and only if each open cover of $X$, of cardinality at most $\mathfrak{m}$, has a finite subcover. $X$ is called $\mathfrak{m}$-quasicompact [9] if and only if each open cover $u$ of $X$ by cozero sets, with the cardinality of $u$ at most $\mathfrak{m}$, has a finite subcover. $X$ is called $\mathfrak{m}$-pseudocompact [5] if and only if each map (continuous function) $f: X \rightarrow R^{\mathfrak{m}}$ has a compact range, where $R^{\mathfrak{m}}$ denotes the Cartesian product of the reals $\mathfrak{m}$ times.

It is clear that every $\mathfrak{m}$-compact space is both $\mathfrak{m}$-pseudocompact and $m$-quasicompact. It is also known that a completely regular, Hausdorff space is m-quasicompact if and only if it is m-pseudocompact [5, Theorem 2.2].

Lemмa 3. Every $\mathfrak{m}$-bounded space is $\mathfrak{m}$-compact.

Proof. Suppose $X$ is an $m$-bounded space which is not m-compact. Then there is an open cover $u=\left\{U_{\alpha} \mid \alpha \in \Lambda\right\}$ with $|\Lambda| \leqq m$ having no finite subcover. Let $\Lambda^{F}$ denote the collection of finite subsets of $\Lambda$.

For each $\gamma \in \Lambda^{F}$, choose $x_{\gamma} \in X \backslash \cup\left\{U_{\alpha} \mid \alpha \in \gamma\right\}$. Then, since $\left|\Lambda^{F}\right|$ $\leqq \mathfrak{m}, A=\left\{x_{\gamma} \mid \gamma \in \Lambda^{F}\right\}$ is a subset of $X$ of cardinality at most $\mathfrak{m}$. Since $X$ is m-bounded, there is a compact set $C \subseteq X$ such that $A \subseteq C$.

Since $u$ covers $C$ there is a finite set $\gamma_{0} \in \Lambda^{F}$ such that

$$
C \subseteq \cup\left\{U_{\alpha} \mid \alpha \in \gamma_{0}\right\} \text {. }
$$

Hence $x_{\gamma_{0}} \notin A$. This is clearly impossible.

Definition 6. Let $X$ be a topological space, let $x \in X$, and let $\mathfrak{m}$ be an infinite cardinal. $x$ is called an $\mathfrak{m}$-point if, for each collection $u$ of open subsets of $X$ satisfying $|u| \leqq m$ and $x \in \cap u$, there is an open set $V \subseteq X$ such that $x \in V \subseteq \cap u$.

Theorem 2. Let $Y$ be a compact $T_{1}$ space, and let $X \subseteq Y$. If each point of $Y \backslash X$ is an $\mathrm{m}$-point of $Y$, then $X$ is $\mathrm{m}$-bounded. 
Proof. Suppose $A=\left\{a_{\alpha} \mid \alpha \in \Lambda\right\} \subseteq X$ and $|\Lambda| \leqq m$. Let $y \in Y \backslash X$. Since $Y$ is $T_{1}$, for each $\alpha \in \Lambda$ there is an open set $U_{\alpha}$ in $Y$ such that $y \in U_{\alpha}$ and $x_{\alpha} \notin U_{\alpha}$. Since $y$ is an m-point there is an open set $U_{\nu}$ of $Y$ such that $y \in U_{y} \subseteq \bigcap\left\{U_{\alpha} \mid \alpha \in \Lambda\right\}$. Let $U=\bigcup\left\{U_{\nu} \mid y \in Y \backslash X\right\}$. Then $U$ is open in $Y$ and $Y \backslash X \subseteq U$. Thus $Y \backslash U=X \backslash U$, and since $Y \backslash U$ is closed in $Y$, it is compact. Hence $X \backslash U$ is compact in $X$, and $A \subseteq X \backslash U$. Thus $X$ is $\mathrm{m}$-bounded.

THEOREM 3. Let $X$ be a linearly ordered space, and let $\mathfrak{m}$ be an infinite cardinal. The following are then equivalent:

(a) $X$ is $\mathfrak{m}$-bounded.

(b) $X$ is m-compact.

(c) $X$ is $\mathfrak{m}$-pseudocompact.

(d) $X$ is $\mathfrak{m}$-quasicompact.

(e) For each gap $u$ of $X,\left|\omega_{\alpha(u)}\right|>\mathfrak{m}$ and $\left|\omega_{\beta(u)}\right|>\mathfrak{m}$.

Proof. We have already seen that $(\mathrm{a}) \Rightarrow(b) \Rightarrow(c)$. Since every linearly ordered space is normal and Hausdorff $(c) \Leftrightarrow(d)$.

That $(\mathrm{e}) \Rightarrow(\mathrm{a})$ is a consequence of Theorem 2 , since, if each gap $u$ of $X$ satisfies $\left|\omega_{\alpha(u)}\right|>\mathfrak{m}$ and $\left|\omega_{\beta(u)}\right|>\mathfrak{m}$, then $u$ is an $\mathfrak{m}$-point in $X^{+}$. Since $X^{+}$is compact and Hausdorff, $X$ is m-bounded. Thus it is sufficient to show $(\mathrm{d}) \Rightarrow(\mathrm{e})$.

Suppose $u$ is a gap of $X$ and $\left|\omega_{\alpha(u)}\right| \leqq \mathfrak{m}$. Then there is a strictly increasing sequence $\left\{x_{\gamma} \mid \gamma<\omega_{\alpha(u)}\right\}$ in $X$ that is cofinal in $\{x \in X \mid x<u\}$. Since $X$ is normal, for each $\gamma<\omega_{\alpha(u)}$ there is a map $f_{\gamma}: X \rightarrow R$ such that $f_{\gamma}(x)=1$ for $x \leqq x_{\gamma}, f_{\gamma}(x)=0$ for $x \geqq x_{\gamma+1}$, and $0 \leqq f_{\gamma} \leqq 1$. Since $u$ is a gap of $X$ there is a map $f: X \rightarrow R$ such that $f(x)=1$ for $x>u$ and $f(x)=0$ for $x<u$.

For each $\gamma<\omega_{\alpha(u)}$ let $U_{\gamma}=\left\{x \in X \mid f_{\gamma}(x) \neq 0\right\}$, and let $U$ $=\{x \in X \mid f(x) \neq 0\}$. Then $\mathcal{u}=\{U\} \cup\left\{U_{\gamma} \mid \gamma<\omega_{\alpha(u)}\right\}$ is an open cover of $X$ by cozero sets and $|u| \leqq m$. Clearly $u$ has no finite subcover, and hence $X$ is not $\mathfrak{m}$-quasicompact.

The following lemma leads to an interesting corollary of Theorem 3 .

Lemma 4. Let $\mathfrak{m}$ be an infinite cardinal, and let $\left\{X_{\alpha} \mid \alpha \in \Lambda\right\}$ be a collection of $\mathrm{m}$-bounded topological spaces. Then $\Pi\left(X_{\alpha} \mid \alpha \in \Lambda\right)$ is $\mathfrak{m}$-bounded.

Proof. Let $X=\Pi\left(X_{\alpha} \mid \alpha \in \Lambda\right)$ and $A \subseteq X,|A| \leqq \mathfrak{m}$. Then $\left|\pi_{\alpha}[A]\right|$ $\leqq \mathfrak{m}$ for each $\alpha \in \Lambda$, where $\pi_{\alpha}: X \rightarrow X_{\alpha}$ is the projection function. Since $X_{\alpha}$ is m-bounded, there is a compact set $C_{\alpha} \subseteq X_{\alpha}$ such that $\pi_{\alpha}[A]$ $\subseteq C_{\alpha}$. Hence $A \subseteq \Pi\left(C_{\alpha} \mid \alpha \in \Lambda\right) \subseteq X$, and $\Pi\left(C_{\alpha} \mid \alpha \in \Lambda\right)$ is compact. 
Corollary. Let $\mathfrak{m}$ be an infinite cardinal, let $\left\{X_{\alpha} \mid \alpha \in \Lambda\right\}$ be a collection of linearly ordered spaces, and let $X=\Pi\left(X_{\alpha} \mid \alpha \in \Lambda\right)$. The following are then equivalent:

(a) $X$ is $\mathfrak{m}$-bounded.

(b) $X$ is m-compact.

(c) $X$ is $\mathfrak{m}$-pseudocompact.

(d) $X$ is $\mathfrak{m}$-quasicompact.

(e) $X_{\alpha}$ is m-bounded for each $\alpha \in \Lambda$.

Proof. As before (a) $\Rightarrow(b) \Rightarrow(c)$, and (c) $\Leftrightarrow(d)$ since $X$ is completely regular and Hausdorff. Also (c) $\Rightarrow(\mathrm{e})$, since any continuous image of an $\mathfrak{m}$-pseudocompact space is $\mathfrak{m}$-pseudocompact. That $(\mathrm{e}) \Rightarrow(\mathrm{a})$ follows from Lemma 4.

\section{REFERENCES}

1. G. Aquaro, Intorno ad una generalizzazione degli spazi paracompatti, Atti Accad. Naz. Lincei Rend. Cl. Sci. Fis. Mat. Natur (8) 38 (1965), 824-827. MR 33 \#3249.

2. V. V. Fedorcuk, Ordered sets and the product of topological spaces, Vestnik Moskov. Univ. Ser. I Mat. Meh. 21 (1966), no. 4, 66-71. (Russian) MR 34 \#3515.

3. L. Gillman and M. Henriksen, Concerning rings of continuous functions, Trans. Amer. Math. Soc. 77 (1954), 340-362. MR 16, 156.

4. S. L. Gulden, Equivalent forms of m-paracompactness, Prace Math. 11 (1968), 265-278. MR 37 \#2182.

5. J. F. Kennison, m-pseudocompactness, Trans. Amer. Math. Soc. 104 (1962), 436-442. MR $26 \# 3009$.

6. M. J. Mansfield, Some generalizations of full normality, Trans. Amer. Math. Soc. 86 (1957), 489-505. MR 20 \#273.

7. K. Morita, Paracompactness and product spaces, Fund. Math. 50 (1961/62), 223-236. MR 24 \#A2365.

8. J. Novak, On the cartesian product of two compact spaces, Fund. Math. 40 (1953), 106-112. MR 15, 640.

9. H. Poppe, Eine Charakterisierung m-quasikompakter Räume, Math. Nachr. 29 (1965), 247-253. MR 32 \#429.

LEHIGH UNIVERSITY

State University of New York at Buffalo and University of Saskatchewan, Regina Campus 